\title{
Presencia de Pseudacysta perseae (Heidemann, 1908) (Insecta: Hemiptera: Tingidae) en Panamá
}

\author{
Presence of Pseudacysta perseae (Heidemann, 1908) \\ (Insecta: Hemiptera: Tingidae) in Panama
}

\author{
Anovel Barba A ${ }^{1,2}$, Vidal Aguilera Cogley², José Ángel Herrera Vásquez ${ }^{2 *}$
}

\section{RESUMEN}

El aguacate (Persea americana Mill), por sus cualidades nutricionales, ha incrementado su uso en Panamá. Sin embargo, los árboles de aguacate se han visto afectados por la destrucción de tejidos en el haz y el envés de las hojas de las plantas dañadas. Esto se refleja en áreas cloróticas visibles en el haz, y las hojas maduras son las preferidas por el insecto para su alimentación. El propósito de la investigación fue determinar el agente causal del daño en árboles de aguacate. Se realizó un muestreo en 28 localidades en la península de Azuero, Panamá, durante el periodo de 2017 a 2019. Se cita por primera vez al insecto Pseudacysta perseae (Heidemann, 1908) (Hemiptera: Tingidae) como plaga del aguacate en Panamá y se determina su distribución en la península de Azuero.

Palabras clave: aguacate, chinche de encaje, nuevo registro, Persea americana, plaga.

\begin{abstract}
The avocado (Persea americana Mill), due to its nutritional and nutritional qualities, has increased its use in Panama. However, avocado trees have been affected by the destruction of tissues in the bundle and on the underside of the leaves of the affected plants, which is reflected in visible chlorotic areas in the bundle, with mature leaves being preferred by the insects for food.

The purpose of the investigation was to determine the causative agent of damage in avocado trees. Sampling was carried out in 28 locations in the Azuero Peninsula, Panama, during the period from 2017 to 2019. The insect Pseudacysta perseae (Heidemann, 1908) (Hemiptera: Tingidae) is cited for the first time, as an avocado pest in Panama and its distribution in the Azuero Panama region is determined.

Keywords: avocado, lacewing bug, new record, Persea americana, pest.
\end{abstract}

\section{Introducción}

El aguacate (Persea americana Mill) ha incrementado su uso en el país por sus cualidades nutricionales. Las variedades Simmonds, choquete, booth, mantequilla y aguacate de leche son las más cultivadas. Se estima que en Panamá se cultivan aproximadamente $572 \mathrm{ha}^{-1}$, con una producción cercana a las 8399 toneladas (FAO, 2018), y el 95\% proviene de la provincia de Chiriquí.

Pseudacysta perseae (Heidemann, 1908) (Hemíptera: Tingidae), conocida frecuentemente como "chinche de encaje del aguacate", es una plaga foliar del aguacate, identificada por primera vez en el sur de la Florida, Estados Unidos de América, en 1908 (Peña et al., 1998; Gagné et al., 2008, EPPO, 2020). Actualmente, la presencia de la especie se reporta en México, Bermuda, Cuba, República Dominicana, Guadalupe, Jamaica, Martinica, Puerto Rico, San Cristóbal y Nieves, Santa Lucía, Trinidad y Tobago, Islas Vírgenes y Venezuela, donde causa graves daños al cultivo de aguacate (Amaraguel et al., 1999; Peña et al., 2012, EPPO, 2020).

Los daños ocasionados se observan en fase de vivero y plantaciones en producción, provocando la destrucción de tejidos en el haz y el envés de las hojas de las plantas afectadas. Esto se refleja

\footnotetext{
1 Laboratorio de Entomología, Centro Regional Universitario de Azuero (CRUA), Universidad de Panamá (UP), Chitré, Herrera, Panamá.

2 Grupo de Investigación de Protección Vegetal (GIPV), Centro de Investigación Agropecuaria Divisa (CIAD), Instituto de Investigación Agropecuaria de Panamá (IDIAP), Ctra. Panamericana, Los Canelos, Santa María, Estafeta de Divisa, 0619 Herrera, Panamá.

* Autor para correspondencia: jose.herrera@idiap.gob.pa; joshervs11@gmail.com
} 
en áreas cloróticas visibles en el haz, y las hojas maduras son las preferidas por el insecto para su alimentación (Mead y Peña, 1998; De la Torre et al., 1999). Estos daños facilitan la entrada del hongo Colletotrichum gloesporiodes (Penz) Penz\&Sacc causante de la antracnosis (Peña et al., 2012). Adicionalmente, ataques severos de esta plaga podrían ocasionar una defoliación completa de la planta (EPPO, 2020). En Cuba, este insecto se considera la principal plaga del cultivo de aguacate, y causa pérdidas de rendimiento superiores al 50\% (Morales et al., 2012), requiriendo control químico cuando los daños foliares superan el 30\%.

El ciclo de vida del chinche de encaje en plantas de aguacate es de 22 días, desde la etapa de huevo hasta la etapa de adulto, pasando por cuatro etapas ninfales (IF-IFAS, 2020). Sus huevos están cubiertos por una sustancia oscura y pegajosa secretada por los adultos, mientras que las ninfas se alimentan succionando los jugos nutritivos de las células de la hoja, en las proximidades del lugar donde se depositaron los huevos (IF-IFAS, 2020).

En Panamá, el aguacate se cultiva en huertos familiares localizados en la periferia de las casas de los agricultores, y su producción está destinada al consumo interno. En este sentido, los productores manifiestan su preocupación por los daños que se presentan en sus plantas, caracterizados por áreas cloróticas y marrones necróticas en las hojas. Este estudio se realizó con el objetivo de determinar la presencia/ausencia y distribución del chinche de encaje en cultivos de aguacate en Panamá.

\section{Materiales y métodos}

Se realizaron muestreos durante el periodo del 7 de junio de 2016 al 27 de julio de 2019 en 28 localidades de las provincias de Coclé, Herrera, Los Santos y Veraguas (Tabla 1). Estas provincias se caracterizan por presentar un clima tropical de

Tabla 1. Localidades donde se recolectaron las muestras de hojas de aguacate en las provincias de Coclé, Herrera, Los Santos y Veraguas (periodo 2016-2019).

\begin{tabular}{|c|c|c|c|c|c|}
\hline \multirow{2}{*}{ Muestra } & \multirow{2}{*}{ Fecha de colecta } & \multirow{2}{*}{ Localidad Corregimiento } & \multicolumn{2}{|c|}{ Coordenadas UTM } & \multirow{2}{*}{ Altura (MNSN) } \\
\hline & & & Longitud Norte & Longitud Oeste & \\
\hline 1 & 7-jun-2016 & Santiago & $8^{\circ} 05^{\prime} 33^{\prime \prime}$ & $80^{\circ} 58^{\prime} 34^{\prime \prime}$ & 90 \\
\hline 2 & 23-jun-2016 & Valle Rico & $7^{\circ} 36^{\prime} 05^{\prime \prime}$ & $80^{\circ} 22^{\prime} 16^{\prime \prime}$ & 230 \\
\hline 3 & 23-jun-2016 & Valle Rico & $7^{\circ} 36^{\prime} 05^{\prime \prime}$ & $80^{\circ} 22^{\prime} 16^{\prime \prime}$ & 230 \\
\hline 4 & 23-jun-2016 & Valle Rico & $7^{\circ} 36^{\prime} 05^{\prime \prime}$ & $80^{\circ} 22^{\prime} 16^{\prime \prime}$ & 230 \\
\hline 5 & 5-jul-2016 & Santiago & $8^{\circ} 06^{\prime} 26^{\prime \prime}$ & $80^{\circ} 58^{\prime} 39^{\prime \prime}$ & 100 \\
\hline 6 & 3-ago-2016 & Las Matas & $8^{\circ} 28^{\prime} 28^{\prime \prime}$ & $80^{\circ} 05^{\prime} 46^{\prime \prime}$ & 170 \\
\hline 7 & 3 -ago-2016 & Las Matas & $8^{\circ} 28^{\prime} 25^{\prime \prime}$ & $80^{\circ} 05^{\prime} 46^{\prime \prime}$ & 170 \\
\hline 8 & 3 -ago-2016 & Las Matas & $8^{\circ} 28^{\prime} 25^{\prime \prime}$ & $80^{\circ} 05^{\prime} 46^{\prime \prime}$ & 170 \\
\hline 9 & 3 -ago-2016 & Las Matas & $8^{\circ} 28^{\prime} 30^{\prime \prime}$ & $80^{\circ} 05^{\prime} 46^{\prime \prime}$ & 200 \\
\hline 10 & 24-ago-2016 & La Soledad & $7^{\circ} 53^{\prime} 01^{\prime \prime}$ & $81^{\circ} 15^{\prime} 57^{\prime \prime}$ & 30 \\
\hline 11 & 24-ago-2016 & Quebrada Grande & $7^{\circ} 43^{\prime} 35^{\prime \prime}$ & $81^{\circ} 22^{\prime} 01^{\prime \prime}$ & 10 \\
\hline 12 & 24-ago-2016 & Quebrada Grande & $7^{\circ} 43^{\prime} 35^{\prime \prime}$ & $81^{\circ} 22^{\prime} 01^{\prime \prime}$ & 10 \\
\hline 13 & 6 -oct-2016 & Los Llanos & $7^{\circ} 57^{\prime} 42^{\prime \prime}$ & $80^{\circ} 50^{\prime} 48^{\prime \prime}$ & 170 \\
\hline 14 & 6 -oct-2016 & Los Llanos & $7^{\circ} 57^{\prime} 42^{\prime \prime}$ & $80^{\circ} 50^{\prime} 48^{\prime \prime}$ & 170 \\
\hline 15 & 6 -oct-2016 & Los Llanos & $7^{\circ} 57^{\prime} 42^{\prime \prime}$ & $80^{\circ} 50^{\prime} 48^{\prime \prime}$ & 170 \\
\hline 16 & 6 -oct-2016 & Los Llanos & $7^{\circ} 53^{\prime} 5^{\prime \prime}$ & $80^{\circ} 53^{\prime} 9^{\prime \prime}$ & 150 \\
\hline 17 & 27-oct-2016 & El Pajonal & $8^{\circ} 37^{\prime} 6^{\prime \prime}$ & $80^{\circ} 14^{\prime} 56^{\prime \prime}$ & 270 \\
\hline 18 & 27-oct-2016 & Toabré & $8^{\circ} 38^{\prime} 4^{\prime \prime}$ & $80^{\circ} 18^{\prime} 15^{\prime \prime}$ & 210 \\
\hline 19 & 6-oct-2016 & Los Llanos & $7^{\circ} 53^{\prime} 5^{\prime \prime}$ & $80^{\circ} 53^{\prime} 15^{\prime \prime}$ & 230 \\
\hline 20 & 6 -oct-2016 & Los Llanos & $7^{\circ} 57^{\prime} 38^{\prime \prime}$ & $80^{\circ} 50^{\prime} 56^{\prime \prime}$ & 160 \\
\hline 21 & 14-dic-2016 & La Espigadilla & $7^{\circ} 53^{\prime} 8^{\prime \prime}$ & $80^{\circ} 23^{\prime} 23^{\prime \prime}$ & 50 \\
\hline 22 & 14-dic-2016 & Guararé & $7^{\circ} 47^{\prime} 34^{\prime \prime}$ & $80^{\circ} 24^{\prime} 30^{\prime \prime}$ & 40 \\
\hline 23 & 14-dic-2016 & Llano Abajo & $7^{\circ} 47^{\prime} 17^{\prime \prime}$ & $80^{\circ} 24^{\prime} 58^{\prime \prime}$ & 70 \\
\hline 24 & 14-dic-2016 & Llano Abajo & $7^{\circ} 45^{\prime} 60^{\prime \prime}$ & $80^{\circ} 24^{\prime} 44^{\prime \prime}$ & 160 \\
\hline 25 & 22-jul-2017 & San Juan Bautista & $7^{\circ} 57^{\prime} 48^{\prime \prime}$ & $80^{\circ} 26^{\prime} 24^{\prime \prime}$ & 35 \\
\hline 26 & 23-jul-2017 & Llano de la Cruz & $7^{\circ} 57^{\prime} 28^{\prime \prime}$ & $80^{\circ} 38^{\prime} 25^{\prime \prime}$ & 44 \\
\hline 27 & 27-jul-2017 & Guararé & $7^{\circ} 47^{\prime} 29^{\prime \prime}$ & $80^{\circ} 16^{\prime} 28^{\prime \prime}$ & 49 \\
\hline 28 & 25-jul- 2019 & El Ejido & $7^{\circ} 54^{\prime} 39^{\prime \prime}$ & $80^{\circ} 22^{\prime} 05^{\prime \prime}$ & 28 \\
\hline
\end{tabular}


sabana, con períodos secos que van de enero a abril y precipitaciones de mayo a diciembre, con un promedio anual de $1300 \mathrm{~mm}$. La elevación de estas regiones geográficas varía entre 0 y $300 \mathrm{msnm}$ (metros sobre el nivel del mar) (ANAM, 2010). La georreferenciación de los huertos se realizó con la ayuda de un sistema de posicionamiento global (GPS) portátil, obteniendo datos de latitud, longitud y altitud, los cuales se registraron en el programa Microsoft Excel® 2013 y se mapearon con el programa QGIS ver 2.18.

Se recolectaron hojas de aguacate a partir de plantas que mostraron síntomas asociados a la presencia del chinche de encaje, tales como decoloración en el haz de las hojas, decoloración y mancha marrón en el envés y defoliación de las plantas (Figura 2).

Las muestras de hojas se colocaron en bolsas plásticas para su traslado al Laboratorio de Entomología del Centro Regional Universitario de Azuero (CRUA), Universidad de Panamá, localizado en Chitré, Herrera, Panamá. Se examinó el número de huevos, ninfas y adultos del insecto en un microscopio de disección Leica M125 (100 x de aumento), cámara DMC 2900 e iluminación LED 5000 SLI, mientras que su procesamiento se realizó con la ayuda del software Leica Application Suite (LAS). La identificación se hizo siguiendo los caracteres descritos por Sandoval y Cermeli (2005). Los insectos se depositaron en la colección de Entomología del Laboratorio de Protección Vegetal (LPV) del Centro de Investigación Agropecuaria Divisa (CIAC), Instituto de Investigación Agropecuaria de Panamá (IDIAP), ubicado en Los Canelos, Santa María, Herrera, Panamá.

\section{Resultados y discusión}

\section{Localización geográfica}

Se inspeccionaron 647 hojas de aguacate para determinar la presencia/ausencia de $P$. perseae. Se identifica la presencia de $P$. perseae en agroecosistema de aguacate, en Panamá, Herrera, San Juan Bautista, 14 ๆ, 2 ○, 22.vii.2017., Herrera, Llano de la Cruz, 3 \&, 23.vii.2017, Los Santos, Guararé, 2 ㅇ, 27.vii.2017, Los Santos, El Ejido, 10 웅 2 ऽ 25.vii.2019 (Cuadro 1) (Figura 1). Los insectos se encontraron en plantas entre 1 y 3 años de edad, a una altura de 28 a $49 \mathrm{msnm}$. No se determinó su presencia en las provincias de Coclé y Veraguas.

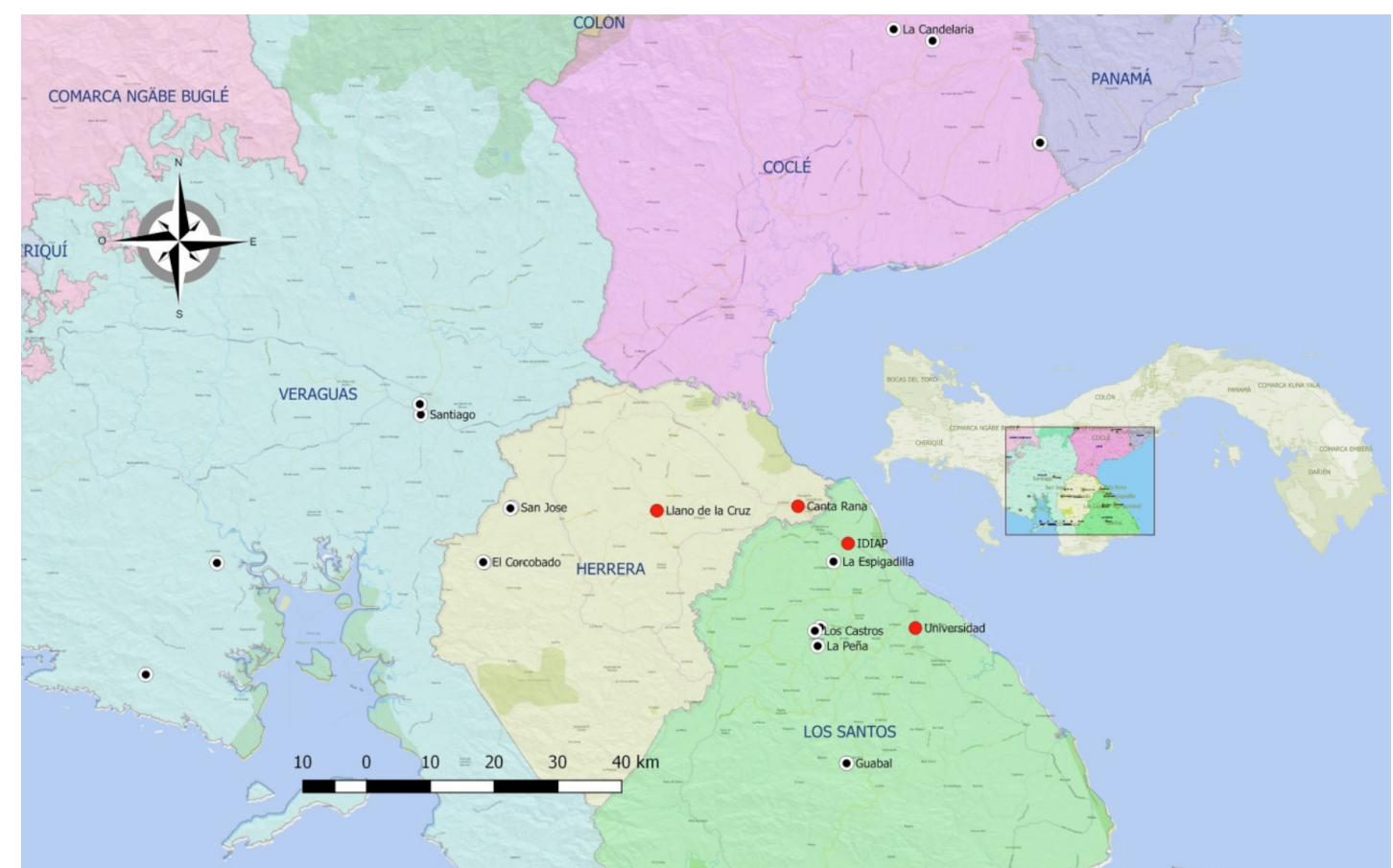

Figura 1. Localización geográfica del área muestreada en las provincias de Coclé, Herrera, Los Santos y Veraguas, Panamá (periodo 2016-2019). Las localidades donde se determinó la presencia de Pseudacysta persae se encuentran marcadas con un círculo de color rojo. 
La importancia de estos hallazgos radica en que son los primeros registros logrados en Panamá. Se trata de una plaga importante, dado que causa defoliación en plantas de aguacate y además existe la posibilidad de que $P$. perseae facilite la penetración de antracnosis (Colletotrichum gloeosporioides) al producir daños físicos en la epidermis de las hojas (Peña et al., 1998). Esto podría afectar los rendimientos del cultivo de aguacate. El insecto solo ha logrado dispersarse en 4 localidades en la región de Azuero.

\section{Descripción del daño}

Los ejemplares de $P$. perseae identificados en este estudio se recolectaron sobre hojas de aguacate que mostraban síntomas asociados a los daños causados en el haz y el envés. En este sentido, las hojas lesionadas presentaron necrosis foliar, mientras que las plantas mostraron defoliación parcial (Figura 2). Los daños de $P$. perseae fueron observados en la zona media inferior de las plantas de aguacate. Se ha determinado que este insecto puede encontrarse y sobrevivir en hojas caídas y luego atacar hojas nuevas (Morales et al., 2012). Sin embargo, no se observó en muestras de hojas recogidas del suelo durante el periodo de estudio.

Las plantas de aguacate presentaron afectaciones que fluctuaron entre 10 y $50 \%$ del área foliar en árboles de 3 años de edad. Se detectó la presencia de todos los estadios del insecto en el envés de las hojas (Figura 3). Los huevos son colocados y observados, dispuestos en hileras o en pequeños grupos, que aparecían cubiertos por las excretas. Según Malumphy

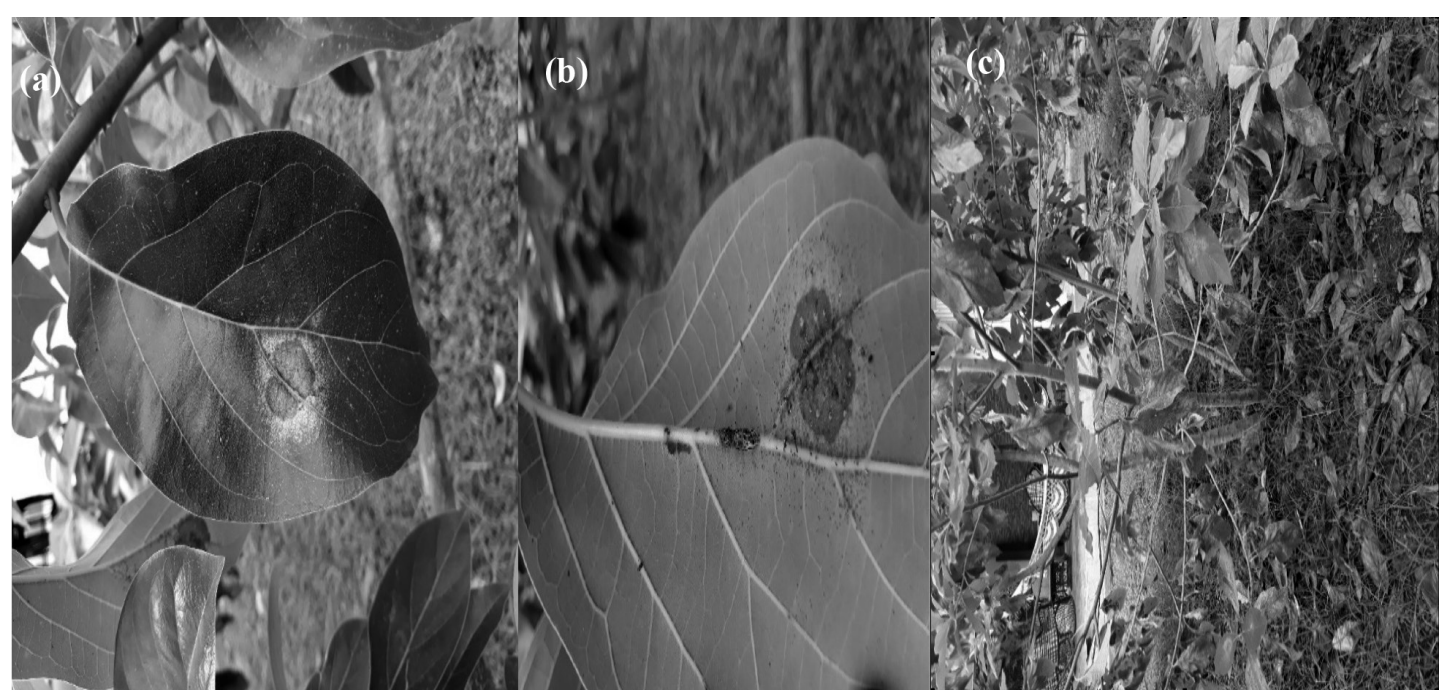

Figura 2. Síntomas de decoloración en el haz de una hoja (a), decoloración y mancha marrón en el envés de una hoja (b) y defoliación parcial de una planta de aguacate (c).

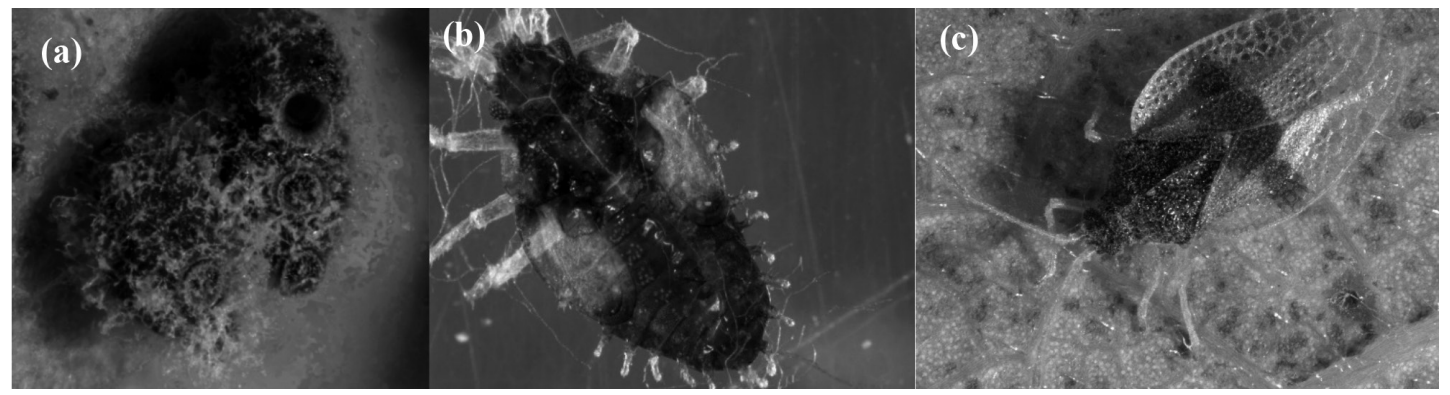

Figura 3. Diferentes estadios biológicos de P. perseae sobre hojas de aguacate: huevos (a), ninfa (b) y adulto. 
(2015), esto parece protegerlos de la desecación y de los enemigos naturales.

Los adultos de $P$. perseae pueden volar por sí solos, pero también se transportan por el viento. No obstante, existe poca información sobre su dispersión natural ya que este insecto no se alimenta de frutos de aguacate. Por ello, parece poco probable su dispersión a través de esta vía, sino que se asocia a las diversas actividades humanas, como la comercial y agrícola (EPPO, 2020).

Durante décadas, $P$. perseae se consideró un insecto plaga de poca importancia económica en el cultivo de aguacate (Peña et al., 2012). Sin embargo, actualmente se reporta como una de las principales plagas que afectan a este cultivo (Morales et al., 2012; Peña et al., 1998). En 2014, se detecta la presencia de este insecto en huertos comerciales en Madeira, Portugal, por lo cual, fue añadido a la lista de alerta de la Organización Europea y Mediterránea de Protección de las Plantas (EPPO) (EPPO, 2020).

\section{Conclusiones}

Se registra por primera vez Pseudacysta perseae en República de Panamá. Se trata de cuatro localidades de la región de Azuero. La presencia de esta nueva especie es de gran importancia para el país, a fin de lograr su erradicación y evitar su dispersión en otras regiones donde no está. Es necesario ampliar la vigilancia fitosanitaria a otras zonas del país.

\section{Agradecimientos}

Los autores expresan su agradecimiento al Lic. I. Zachrisson (CIAD, IDIAP, Panamá) por su asistencia técnica en el mapeo de las coordenadas UTM. Al ingeniero Orlando Osorio, por revisión del manuscrito. De igual forma, agradecen al Lic. Andrés Salazar, especialista en productos Alpha Mediq S.A., por su apoyo en microscopía. El Dr. J.A. Herrera-Vázquez fue apoyado por el Sistema Nacional de Investigación (SNI, Panamá) de la Secretaría Nacional de Ciencia, Tecno logía e Innovación (SENACYT, Panamá).

\section{Literatura Citada}

Almaguel, L.; Blanco, E.; Suárez, P.; De La Torre, P.; Cáceres, I.; Nieves, C.; Márquez, M.; Blanco, L.

1999. Control de la chinche del aguacate (Pseudacysta perseae (Heidemann)) en ciudad de La Habana. Fitosanidad, 3(2): 69-74.

ANAM.

2010. Atlas ambiental de la República de Panamá: Características físicas. Autoridad Nacional del Ambiente. Panamá. 188 p.

De La Torre, P.; Almaguel, L.; Blanco, E.

1999. Daños, distribución y enemigos naturales de la chinche de encaje del aguacate Pseudacysta perseae (Heidemann) (Hemiptera:Tingidae). Fitosanidad, 3(2): 65-67. EPPO.

2020. Pseudacysta perseae (Hemiptera: Tingidae). European and Mediterranean Plant Protection Organization. Disponible: https://gd.eppo.int/reporting/article-4365. FAO. Consultado:27/abril/ 2020.

2018. Producción de cultivo de aguacate, Panamá. Organización de las Naciones Unidas para la Agricultura y la Alimentación. Disponible: http://www.fao.org/faostat/es/\#data/QC. Consultado: 30/oct/2018.

Gagné, R.J.; Peña, J.E. y Acevedo F.

2008. A new Lestodiplosine (Diptera: Cecidomyiidae) preying on the avocado lace bug, Pseudacysta perseae (Heteroptera: Tingidae) in Southern Florida. Florida Entomologist, 91 (1): 43-48.

Malumphy, C.

2015. Avocado lace bug Pseudacysta perseae. The Food and Environment Research Agency. Sand Hutton, York, UK. Disponible: www.fera.defra.gov.uk. Consultado: 29/oct/2018.
Mead, F.W.; Peña, J.E.

1998. Avocado lace bug, Pseudacysta perseae (Heidemann) (Insecta: Hemiptera: Tingidae). Florida Dep Agr \& Cons Serv Div Plant Indus. Entomology Circular, 346. Disponible: https://edis.ifas.ufl.edu/pdffiles/IN/IN16600. pdf. Consultado:27/abril/2020

Morales, R.L.; Grillo, R.H.; Maza, E.N.; Grau, R.

2012. Efectividad de hongos entomopatógenos en el manejo de Pseudacysta perseae (Heid.) (Hemiptera: Tingidae) en aguacate (Persea americana Mill.). Revista Científica UDO Agrícola, 12 (3): 599-608.

Peña, J.E.; Duncan, R.E.; Roltsch, W.J.; Carrillo, D.

2012. Mortality factors of the avocado lace bug, Pseudacysta perseae (Heteroptera: Tingidae), in Florida. Florida Entomologist, 95(1): 179-182.

Peña, J.E.; Sundhari, S.; Duncan, R.; Shaffer, B.

1998. Monitoring, damage, natural enemies and control of avocado lace bug, Pseudacysta perseae (Hemiptera: Tingidae). Proceedings of the Florida State Horticultural Society, 111: 330-334.

Sandoval, M.F.; Cemeli, M.

2005. Presencia de Pseudacysta perseae (Heidemann, 1908) (Insecta: Hemiptera: Tingidae) en Venezuela. Entomotropica, 20(3): 271-273.

UF-IFAS.

2020. Featured creatures, Avocado lace bug Pseudacysta perseae (Hiedemann) (Hemiptera: Tingidae). University of Florida. Disponible en: http://entnemdept.ufl.edu/creatures/ fruit/avocado_lace_bug.htm.Consultado: 27/abril/2020. 
